\title{
Essais
}

Revue interdisciplinaire d'Humanités

$11 \mid 2017$

Fictions de l'identité

\section{Aphoristique et identité}

Dialogue de Lia Kurts et François Rastier

\section{Lia Kurts-Wöste}

\section{OpenEdition}

\section{Journals}

Édition électronique

URL : http://journals.openedition.org/essais/3502

DOI : 10.4000/essais.3502

ISSN : 2276-0970

Éditeur

École doctorale Montaigne Humanités

\section{Édition imprimée}

Date de publication : 15 mai 2017

Pagination : 99-107

ISBN : 979-10-97024-01-7

ISSN : 2417-4211

\section{Référence électronique}

Lia Kurts-Wöste, « Aphoristique et identité », Essais [En ligne], 11 | 2017, mis en ligne le 14 octobre 2020, consulté le 21 octobre 2020. URL : http://journals.openedition.org/essais/3502 ; DOI : https:// doi.org/10.4000/essais.3502 


\section{Aphoristique et identité Dialogue de Lia Kurts et François Rastier, suivi d'aphorismes de François Vaucluse}

Lia Kurts-Wöste

À la mémoire de Urs Widmer.

François Rastier : Vous souhaitez publier dans ce recueil, en annexe à notre dialogue introductif, des aphorismes de François Vaucluse : j'admire votre témérité, car cet auteur, peut-être à dessein, reste des plus confidentiels. Je ne me sens guère qualifié pour l'évoquer, sinon que je suis de ses rares lecteurs, et que j'ai étudié jadis l'aphorisme chez Chamfort et la notion - apparemment homonyme - d'aphorisme chez Saussure.

Lia Kurts : Vous vous inscrivez explicitement dans la lignée des écrits de Saussure (mais des écrits autographes, pas du Cours de Linguistique Générale, largement apocryphe, et parfois fautif) : et précisément, si j'ai bien compris (je tente un résumé de votre position), la notion d' " aphorisme » est centrale chez Saussure, puisqu'elle définit tout bonnement sa méthode, et, de manière plus fondamentale encore, son épistémologie. Ses " aphorismes " correspondent de fait aux grandes dualités sur lesquelles est fondée son étude des langues (langue/parole, diachronie/synchronie, singulier/collectif... qui ne sont pas des oppositions, contrairement à ce que le CLG a laissé entendre). Ce sont donc, fidèles en cela à l'étymologie, des "délimitations " (apo-horizein), c'est à dire des dualités de saisie, de point de vue. Elles définissent un mode d'objectivation très spécifique : il n'y a pas dans sa théorie de fondement, ou d'axiome, ou de principe indéterminé, il n'y a qu'une multiplicité de points de vue interdépendants, dont aucun n'est plus fondamental que l'autre et qui sont tous nécessaires pour objectiver les réalités linguistiques. Ce mode d'objectivation s'oppose d'après vous en particulier à l'apodictique d'Aristote (l'évidence par démonstration et déduction) ; chez Saussure, point d'évidence, ni de logique déductive (qui supposerait un principe fondamental). 
Toujours d'après ce que j'ai compris de vos travaux, Saussure, qui est un grand spécialiste du sanscrit, aurait ici une profonde communauté de pensée et de méthode avec certaines théories bouddhiques du langage ; c'est ce qui expliquerait en partie que Saussure ait été largement incompris, trop distant de nos réflexes culturels en matière de définition de la rationalité scientifique et de ses modes d'objectivation.

Ainsi, les aphorismes (?) de F. Vaucluse sont plutôt (en partie au moins) des aphorismes à la manière des moralistes, mais j'ai l'intuition qu'ils sont dans le même temps un hommage indirect au maître genevois. Si celle-ci vous paraît juste, pourriez-vous développer cette idée d'une parenté entre l'aphorisme littéraire et l'aphoristique saussurienne?

F. R. : Je m'interroge sur le substrat ontologique de la proposition déclarative simple, sur laquelle s'est édifiée la tradition de la logique des classes, dont la syllogistique a été, d'Aristote aux Idéologues comme Destutt de Tracy, le domaine privilégié : connaître, c'est classer, donc rapporter une idée à une autre, plus générale, qui l'inclut. Hélas, il en va toujours ainsi dans des domaines comme la représentation des connaissances et le Web sémantique : on multiplie les " ontologies " et l'on maintient la relation prédicative simple A relation B, comme dans les triplets RDF (Resource Description Framework), dont en avril 2012 le promoteur du Web Sémantique, l'illustre Tim BernersLee, s'enorgueillissait de compter 31 milliards. En sémantique, on privilégie l'hypéronymie et hyponymie.

L'antique sentence, la gnomè, énonçait une vérité morale - ce faisant, elle en restait décisoirement à un conformisme jugé positif. Mais elle a toujours été menacée par le paradoxe, si prisé par les sophistes et les cyniques. À leur tour, les mystiques s'en sont emparés, ruinant l'ordre de ce monde pour en évoquer un autre. La théologie négative a développé un antinomisme. Les Schwärmer comme Hammann s'en sont servis, non sans humour, contre le rationalisme kantien. Il revient je crois à Friedrich Schlegel d'avoir conféré au paradoxe anti-sentencieux le ressort d'une métaphysique illuminative : Der Witz ist Blitz.

Comme on le voit avec les énigmes du Chan et les koans du Zen, l'illumination peut naître d'une phrase tellement anticonformiste qu'elle semble absurde et peut déclencher une métanoia radicale. J'ai eu le plaisir de publier à ce propos un article de Simon Bouquet sur les koans : comme il a édité Saussure, j'ai le sentiment que vous brûlez, divinatoirement. Autre indice de la parenté secrète de l'aphorisme littéraire et de l'aphoristique saussurienne, Nunzio La Fauci, un romaniste de Zurich, a publié des Facettes de linguistique saussurienne, recueil d'aphorismes à thème épistémologique (il m'a permis de les rééditer dans Texto !) - il est par ailleurs l'auteur de plusieurs recueils d'aphorismes explicitement littéraires et créateur d'une collection L'isola di Ferdinando, consacrée à Saussure et au saussurisme. 
F. R. : Cependant, l'aphorisme comme genre littéraire bref-et l'aphorisme dans la rare acception étymologique qu'atteste Saussure (une dualité oppositive de points de vue complémentaires) restent homonymes. Pour réduire quelque peu leur distance, on peut cependant rappeler que pour Saussure le sens est fait de différences. C'est ce qui le sépare décisivement de l'ontologie, puisqu'au contraire elle repose tout entière sur le principe d'identité (tel que $\mathrm{A}=\mathrm{A}$ ) et sans doute le titre de l'écrit de Vaucluse que vous avez choisi, $A$ vs $A$ conteste-t-il l'unité et l'identité à soi de l'être - Énoncer est une tautologie, l'on sait avec Wittgenstein que la tautologie est vide de sens. Les tautologies, universellement répandues, du type comme en français Une femme est une femme, ou La guerre c'est la guerre sont parfaitement endoxales, conformistes, voire fatalistes.

Au principe d'identité on peut opposer un principe de différentialité : le principe saussurien $\mathrm{A} \neq \mathrm{B}$, voire $\mathrm{B} \neq \mathrm{B}$ ' (dans l'exemple Messieurs, Messieurs !) : aucune unité linguistique ne se répète à l'identique et toute occurrence est un hapax.

Il faudrait, pour retracer l'histoire de ce problème, revenir aux differentiae des maîtres de la seconde sophistique, aux synonymistes du XVIII' siècle et à l'Abbé Girard établissant qu'il n'y a aucun synonyme en aucune langue, à Schleiermacher affirmant dans son herméneutique que tout mot est un hapax, à Saussure reconnaissant l'impossibilité de la synonymie, jusqu'à Pottier de nos jours.

Cela ne se réduit pas à un problème de linguistique mais intéresse le statut de notre monde sublunaire et notre préconception du réel. Les différences sont caractéristiques du réel mondain : le monde défini par Platon comme la région des dissemblances (Politique, 273d), repris par Plotin (Ennéades, I, 8, 13, 16-17) puis par Augustin (Confessions, VII, 10, 16, «in regione dissimilitudinis»). La création de l'Être supprime les différences ou du moins les rejette comme inessentielles : la distinction classique entre la substance et les accidents introduit une hiérarchie qui prime l'éternité de la substance. Les différences sont accidentelles et n'entrent en rien dans la définition des substances.

L. K. : Ainsi la proposition déclarative et prédicative simple sur laquelle repose la tradition philosophique occidentale de type déductiviste et/ou ontologique combine-t-elle deux présupposés, le second étant la conséquence du premier : l'absence de modalisation - que suppose au contraire le principe de différentialité - et (donc) l'impossibilité de penser une possible métanoïa - qui suppose plusieurs points de vue, une modalisation de la pensée.

F. R. : En effet, l'ontologie est par principe anhistorique !

Or l'édifice ontologique s'effondre quand la physique au tournant des années 1880 détruit la notion de substance. Et dans la même période, les sciences historiques, dont la linguistique que réfléchit Saussure, récusent toute pérennité a priori, alors que les substances sont par définitions pérennes - ce qui les différencie des accidents. 
Ainsi Saussure écrit-il cet aphorisme (il emploie ce mot, à moins que les éditeurs ne le lui aient prêté) qui figure à la dernière page des Écrits de linguistique générale :

${ }^{(3328.2)} \mathrm{XI}$ Dans la langue, aussi bien que dans tout autre système sémiologique, il ne peut pas y avoir de différence entre ce qui caractérise une chose et ce qui la constitue.

Laphorisme - sans nulle prétention littéraire - assume ici sa dimension critique en unifiant ce qui est distingué principiellement par la tradition ontologique, les accidents et la substance. Cela ruine par suite la "différence » fondamentale entre les étants (soumis aux accidents) et l'Être (invariable et identique à lui-même).

L. K. : On sait par ailleurs que Schleiermacher, qui est pour vous l'un des pionniers de ce que vous appelez l'herméneutique matérielle, écrivait lui aussi des aphorismes : si le sens ne se définit que comme différence au sein d'un texte, cela confirme le lien que vous faites entre pluralisation des modes d'objectivation (aphoristique saussurienne) et dé-ontologisation de l'interprétation (herméneutique matérielle).

F. R. : En effet, les caractères sont relatifs : ils ne sont que des différences qualifiées au sein d'un ensemble de comparaison (le genre, l'intertexte, le corpus). Comme cet ensemble n'est nullement donné, les éléments caractérisants procèdent du point de vue qui a présidé à sa constitution, et de la pratique interprétative qui qualifie les différences.

Le point de vue comparatif qui est le nôtre conduit à ne définir l'identité que comme spécificité. Entre des spécificités, il n'y a point de contradiction, mais seulement des différences. On peut établir entre elles une égale distance critique, alors que les identités tendent à s'affirmer comme des tautologies narcissiques. Même dans le cas de l'identité personnelle, la volonté identitaire reste une aliénation, car le Moi n'a pas de centre et ne peut en être un : il se construit en effet dans des pratiques, donc en relation avec des objets et des personnes.

Les idéologies philosophiques identitaires, dans la tradition inaugurée par Heidegger et qui se poursuit chez des islamistes comme Vadillo ou des eurasistes comme Douguine (conseiller de Poutine) ont naturellement récusé les sciences - en même temps que tout principe de réalité. Heidegger entend sauver et refonder la tradition l'ontologie pour l'instrumentaliser en termes de supériorité gnoséologique (la vérité est affaire de Dasein collectif), linguistique (l'allemand est la langue de l'Être) et ethnique (la Rassengedanke l'établit).

Dans un tout autre continent de la pensée, il y aurait beaucoup à dire sur l'essor des ontologies liées notamment au Web sémantique - et dont l'arrièreplan normatif, totalisant, voire totalitaire vient d'être éclairé par la révélation des activités clandestine de la NSA -et de certains géants du Web. 
Dès lors, l'identification d'une unité linguistique ou sémiotique quelconque dépend de son interprétation - alors que dans la tradition ontologique il en va à l'inverse, ce que le positivisme a reconnu comme l'évidence des données. Or la notion même de donnée demeure invalide : du moins, les données sont ce qu'on se donne, voire ce que l'on vous prend (les Big Data, par exemple).

En termes saussuriens, les signes ne sont aucunement donnés, mais doivent être construits un à un par l'interprétation : aussi le saussurisme, par sa portée critique intéresse l'ensemble des sciences de la culture, dont bien entendu la science de la littérature. Je sais bien que l'expression est passée de mode, mais elle n'a nullement épuisé sa mission, et j'espère que la critique littéraire puisse cesser de mixer le coruscant name-dropping et le bon plaisir académique, la poétique à la française de compiler des inventaires de procédés.

L. K. : Et la manière de pratiquer la littérature - ici, le genre aphoristique - pourrait être alors une manière d'être fidèle à une dé-ontologie herméneutique fondée sur un principe critique ? et peut-être même le symptôme d'une conception originale de la scientificité dans les sciences humaines, où toute "donnée " est le résultat d'un parcours interprétatif, donc d'une forme d'approximation?

Lisant le livre de F. Goyet, Les audaces de la prudence, je ne peux que remarquer le lien - qui vient à l'encontre d'une vision scientiste des sciences humaines et en particulier des sciences du langage, vision que vous stigmatiser - entre la définition paradoxale de la prudence chez Aristote puis Montaigne telle qu'elle apparaît dans ce livre, et le principe qui régule à la fois l'aphoristique saussurienne et le genre littéraire de l'aphorisme. La prudence, c'est ce dosage complexe entre une parfaite maîtrise des différents paramètres à prendre en compte dans la décision et le fait d'assumer la part de risque inhérent à la décision. Les situations herméneutiques paraissent de ce point de vue constituer typiquement des situations prudentielles pour l'interprète, où c'est à partir de la reconnaissance de la part d'approximation inhérente à toute posture interprétative que peuvent se construire les séries de critères pour évaluer la qualité de cette approximation. En ce sens, c'est l'illusion d'exactitude qui est nonrigoureuse : on rejoint par-là votre définition, néo-saussurienne, des sciences humaines comme "sciences rigoureuses" et non sciences exactes.

Par ailleurs, le genre littéraire de l'aphorisme semble avoir pour traits définitoires la brièveté (vous le rappeliez avec l'exemple de Schlegel) et un principe régulateur de type téléologique, fondé précisément sur la possibilité d'une métanoïa (radicale ou simplement locale, par " déverrouillage " de la pensée, ou plutôt de ses principes régulateurs). Or, selon la belle formule de F. Goyet, "être prudent, c'est tout dire en un seul mot ".

N'y aurait-il pas alors un lien de continuité entre la reconnaissance de la part d'approximation inhérente aux procédures interprétatives et le choix 
délibéré d'une forme qui, par sa brièveté ( « tout dire en un seul mot », cet «art du peu " que revendique F. Vaucluse, où la modération, comme chez Aristote, est la plus exigeante des disciplines), fonctionne par approximations successives, construisant une posture énonciative prudente qui, à rebours de notre définition moderne de la prudence, ose précisément une pensée risquée, parfois aux limites du pensable (vous parliez de l'absurde). Le genre littéraire de l'aphorisme serait une manière de s'appuyer sur l'idée, paradoxale pour les Modernes et pourtant pensée par les Anciens, que l'approximation est rigueur, du moins lorsqu'elle est critérisée, le genre de l'aphorisme expérimentant ce principe du côté d'une pensée non scientifique, et cependant non contradictoire avec les principes de scientificité présupposés par l'aphoristique saussurienne, cherchant une pensée qu'on pourrait dire essayée, une pensée libre, où le rire a sa part (cf. le Witz de Schlegel) - et les aphorismes de F. Vaucluse me paraissent aller dans le sens de cet "art de sourire " que met en valeur Bruno RogerVasselin dans son livre Montaigne et l'art de sourire à la Renaissance.

F. R. : C'est pourquoi se référer ici aux moralistes serait ici excessif ; mais les enjeux éthiques et politiques de la réflexion valclusienne sur l'identité ne me semblent en rien contradictoires avec la dialectique anti-ontologique (ou dé-ontologique) de Saussure. À ma connaissance, Vaucluse ne le mentionne mais revendique l'usage de l'aphorisme, comme en témoigne une laisse d'aphorismes sur le genre de l'aphorisme parue dans l'édition bilingue procurée par Francesco Caramagna. Aussi a-t-il obligeamment accepté d'en joindre quelques-uns à la séquence anti-identitaire, intitulée $A$ vs $A$ que vous annexez ci-dessous.

On reproche encore à Saussure de n'avoir pas rédigé de manière continue quelque beau gros traité, dont le CLG a fini par prendre la place, sans s'aviser que le genre du traité procède de la tradition ontologique, allant uniment des principes aux corollaires, des causes aux conséquences, du général au particulier, dessinant ainsi une série de subsomptions, une hiérarchie qui se reflète dans son plan.

Pour une pensée dé-ontologique, les distinctions déterminantes ne sont pas des commencements absolus. Pascal avait réfléchi ainsi, de manière critique, l'espace du recueil : "J'écrirai ici mes pensées sans ordre et non pas peutêtre dans une confusion sans dessein. C'est le véritable ordre et qui marquera toujours mon objet par le désordre même. " (Pascal, Pensées, fragment 472).

Dans sa préface à De l'essence double du langage, Saussure écrit : "Il paraît impossible en fait de donner une prééminence à telle ou telle vérité de la linguistique, de manière à en faire le point de départ central : mais il y a cinq ou six vérités fondamentales qui sont tellement liées entre elles qu'on peut partir indifféremment de l'une ou de l'autre et qu'on arrivera logiquement à toutes les autres et à toute l'infime ramification des mêmes conséquences en partant de l'une quelconque d'entre elles. » 
Il abandonne ainsi la pensée hiérarchique, telle que l'exigent les arborescences ontologiques, pour une pensée réticulaire. Il ajoute, dans ses Notes pour un livre sur la linguistique générale (1893-1894) : "Il y a donc véritablement absence nécessaire de tout point de départ, et si quelque lecteur veut bien suivre attentivement notre pensée d'un bout à l'autre de ce volume, il reconnaîtra, nous en sommes persuadé, qu'il était pour ainsi dire impossible de suivre un ordre très rigoureux. Nous nous permettrons de remettre, jusqu'à trois ou quatre fois sous différentes formes, la même idée sous les yeux du lecteur, parce qu'il n'existe réellement aucun point de départ plus indiqué qu'un autre pour y fonder la démonstration. "

Ainsi assistons-nous à une dernière rencontre entre l'aphoristique saussurienne et le genre même du recueil d'aphorismes.

Tout sépare cependant Saussure des sentences des grammairiens antiques. Saussure pratiquait évidemment Panini et la tradition des grammairiens védiques. Mais là où les soutras de ces grammairiens, ramassées et définitives, offraient matière à commentaires pour leur glossateurs et scoliastes, et présentaient des thèses sur un mode dogmatique, (conforme au fondement ontologique de la tradition védique, puisque les Védas expriment des vérités bien entendu éternelles), Saussure crée un mode hypothétique et critique qui le rend encore incompréhensible pour bien des grammairiens.

Le style propre de Saussure, qui transparaît bien peu dans le $C L G$, est fait de précision et de décision : le ductus du geste de pensée me semble exemplaire, et la frappe du propos fait jeu égal avec les meilleurs aphoristes. Certes, on ne loue pas Saussure pour son style, mais en bonne sémiotique, le sens des textes théoriques est inséparable de leur expression.

Alors que notre langue littéraire, sur le modèle des Goncourt, prime l'emphase, les gros adjectifs multicolores, et perd en force ce qu'elle gagne en violence, il manque à notre discours critique une théorie de la concision. La force du langage réside dans l'immensité de ce qu'il évoque, comme dans la sobriété de ce qu'il dit.

En récusant encore sur ce point la philosophie du langage, et en m'inspirant de Saussure, je voudrais souligner que la formation des paradigmes reste caractéristique des langues humaines (alors qu'ils restent étrangers aux systèmes de communication animaux - comme aux systèmes formels, bref aux anges et aux bêtes). Cette énergie paradigmatique participe de la force évocatoire de l'aphorisme : il ne répète pas le connu (ancien ou à venir), mais il ouvre un monde, par un minuscule pertuis.

Dans le chapitre "Aphorismes » des ELG, Saussure écrit :

${ }^{(3328.1)} \mathrm{X}$ La condition de tout fait linguistique est de se passer entre deux termes au minimum ; lesquels peuvent être successifs ou synchroniques. L'absence du second terme, si elle semble où que ce soit se produire, n'est qu'apparente. 
L'absence, et le maniement des entités absentes, passées ou futures, défuntes ou hiératiques, semble le propre de l'homme - qu'il doit au développement providentiel mais inconsidéré de son cortex préfrontal. Ainsi le non-dit reste-il caractéristique de notre communication.

L. K. : On peut lire dans les Écrits de linguistique générale une formule pour le moins surprenante : l'interprétation y est définie par Saussure comme un processus "par inférence et divination ». Outre que la référence aux procédures d' "inférence " signale l'intérêt de Saussure pour le faire interprétatif ou la pragmatique interprétative (traditionnellement associée à Peirce), le mot « divination " choque l'esprit scientiste, et il a en effet quelque chose de provocant - où le sens de l'autodérision n'est peut-être pas absent. Mais il me semble qu'il ne contredit pas, malgré les apparences, la position critique qui est celle d'une linguistique historique et comparée, s'il désigne précisément la part prudentielle du faire interprétatif que l'on vient de mettre en valeur, et l'acceptation d'une part d'intuition dans les procédures interprétatives et scientifiques, insuffisante comme telle, mais pourtant présente.

F. R. : La problématique interprétative est en effet inférentielle : l'indice n'est que la réification d'une chaîne d'inférences — et ce que Ginzburg nomme le paradigme indiciaire privilégie de fait le raisonnement bayésien, fondé sur la plausibilité plus que sur la probabilité.

Deux conceptions de la culture, comme des performances et des objets culturels s'opposent ici, même si par ailleurs elles se complètent : la conception fréquentiste, qui privilégie les phénomènes sériels, et la conception bayésienne, qui privilégie les phénomènes singuliers. La première est nomothétique, et voudrait élever des régularités au rang de lois ; la seconde, idiographique, admet que la généralité n'est pas le seul objet des sciences (comme le prétend le scientisme) mais que les particularités doivent également être décrites. C'est là bien sûr le principe d'une science des ouvres - déjà de longue date reconnu par la tradition philologique, de Valla à Politien, de Frédéric Schlegel à Schleiermacher, de Panofsky à Spitzer et Auerbach, ou aujourd'hui de Segre à Ginzburg.

La philologie des textes saussuriens ne fait pas exception... Les notes regroupées par Saussure sous le titre Aphorismes restent à interpréter au sein du dossier génétique de son livre inachevé. Il n'est pas rare en effet, dans de tels dossiers, que des passages séminaux figurent isolés. Ils sont ensuite réélaborés dans le contexte auquel ils ont donné naissance.

Prenons donc garde à l'illusion fragmentaire : Héraclite, modèle de l'écriture fragmentaire pour nos contemporains - n'écrivait pas de fragments : son œuvre ne nous est connue que par la doxographie, elle-même souvent fragmentaire (au sens philologique du terme). Un aphorisme de François Vaucluse (il me semble, je cite de mémoire) pose d'ailleurs cette question qu'il attribue 
à un déconstructionniste : "Pourquoi les anciens Grecs écrivaient-ils des fragments ? - Ils habitaient des ruines. »

Les passages isolés dans le dossier génétique peuvent être lus comme des éléments d'une doxographie intime restée inédite. Ils ne relèvent pas du genre de l'aphorisme, mais leur dialectique aphoristique reste (pour nous du moins) une source d'inspiration.

La section Aphorismes des ELG contient une note sur les aphorismes, qui conclut l'ouvrage (du fait des éditeurs) :

${ }^{(3328.5)}$ XIV Quelques vérités qui se retrouvent :

Ne parlons ni d'axiomes, ni de principes, ni de thèses. Ce sont simplement et au pur sens étymologique des aphorismes, des délimitations. [...] mais des limites entre lesquelles se retrouve constamment la vérité, d'où que l'on parte.

Ce pourrait être une conclusion de notre dialogue : l'identité des points de vue serait illusoire, elle ne consiste que dans leur rencontre, témoignage d'une vérité qui doit encore être mise à l'épreuve. Mais ce partage multiplie.

Lia Kurts-Wöste EA 4195 TELEM

Université Bordeaux Montaigne lia.kurts@u-bordeaux-montaigne.fr 